Check for updates

Cite this: Chem. Sci., 2019, 10, 6099

๑ All publication charges for this article have been paid for by the Royal Society of Chemistry

Received 26th March 2019

Accepted 2nd May 2019

DOI: $10.1039 / c 9 s c 01480 b$

rsc.li/chemical-science

\title{
Combination of gallium(III) with acetate for combating antibiotic resistant Pseudomonas aeruginosa $\uparrow$
}

\author{
Yuchuan Wang, (D) $t^{\mathrm{ab}}$ Bingjie Han, (D) $\dot{t}^{\mathrm{a}}$ Yanxuan Xie, $\dot{t}^{\mathrm{a}}$ Haibo Wang, (D) $\dot{t}^{\mathrm{b}}$ \\ Runming Wang, (D) ${ }^{b}$ Wei Xia, (D) ${ }^{a}$ Hongyan $\mathrm{Li}^{\mathrm{D}}{ }^{\mathrm{b}}$ and Hongzhe Sun (iD) $* \mathrm{~b}$
}

\begin{abstract}
Gallium(III) has been widely used as a diagnostic and therapeutic agent in clinics for the treatment of various diseases, in particular, Ga-based drugs have been exploited as antimicrobials to combat the crisis of antimicrobial resistance. The therapeutic properties of Ga(III) are believed to be attributable to its chemical similarity to Fe(III). However, the molecular mechanisms of action of gallium remain unclear. Herein, by integrating metalloproteomics with metabolomics and transcriptomics, we for the first time identified RpoB and RpoC, two subunits of RNA polymerase, as Ga-binding proteins in Pseudomonas aeruginosa. We show that Ga(II) targets the essential transcription enzyme RNA polymerase to suppress RNA synthesis, resulting in reduced metabolic rates and energy utilization. Significantly, we show that exogenous supplementation of acetate could enhance the antimicrobial activity of Ga(III), evidenced by the inhibited growth of persister cells and attenuated bacterial virulence. The effectiveness of co-therapy of $\mathrm{Ga}(\mathrm{III})$ and acetate was further validated in mammalian cell and murine skin infection models, which is attributable to enhanced uptake of Ga(III), and reduced TCA cycle flow and bacterial respiration. Our study provides novel insights into the mechanistic understanding of the antimicrobial activity of $\mathrm{Ga}($ (III) and offers a safe and practical strategy of using metabolites to enhance the efficacy of Ga(III)-based antimicrobials to fight drug resistance.
\end{abstract}

\section{Introduction}

Antimicrobial resistance (AMR) is one of the most serious global public health threats of this century. There is an urgent need to develop safe, efficacious and novel antimicrobial strategies. Owing to the lack of new antibiotics, metal-based antimicrobials have received increasing interest as promising alternatives in recent years for tackling the antimicrobial resistance crisis. ${ }^{1-5}$ Moreover, antimicrobial metals have the capability of disrupting multiple bacterial physiological processes and improving the cure rates of infections from resistant bacterial strains, thus they may also serve as antibiotic adjuvants to restore and boost antibiotic efficacy. ${ }^{2,3,6}$ This is exemplified by our recent study that an antiulcer bismuth drug (CBS, De-Nol®) could be repurposed as an antibiotic adjuvant to treat NDM1-positive bacterial infection in mouse infection models. ${ }^{1}$

Pseudomonas aeruginosa ( $P$. aeruginosa) is a classic opportunistic pathogen, which can cause a variety of hospital-acquired

${ }^{a}$ School of Chemistry, Sun Yat-sen University, Guangzhou, 510275, P. R. China ${ }^{b}$ Department of Chemistry, The University of Hong Kong, Hong Kong, P. R. China. E-mail: hsun@hku.hk

$\dagger$ Electronic supplementary information (ESI) available: Experimental procedures, supplementary figures and omics data. See DOI: 10.1039/c9sc01480b

\$ These authors contributed equally to this work. infections including cystic fibrosis (CF), urinary tract infections (UTIs), skin and pulmonary infections, and even sepsis in severe cases. ${ }^{7,8}$ The infections by $P$. aeruginosa are usually resistant to multiple antibiotics due to the bacterium's intrinsic drug resistance and additional hospital/community acquired resistance. ${ }^{9}$ Gallium compounds such as gallium nitrate, the active component of the FDA-approved formulation Ganite ${ }^{\circledR}$, galliumprotoporphyrin IX, gallium-desferrioxamine show great potential as anti-P. aeruginosa therapeutic agents. ${ }^{10-12}$ Recently, gallium nitrate has been shown to be effective in the treatment of chronic $P$. aeruginosa airway infections both in a mouse infection model and in a phase I clinical trial in individuals with cystic fibrosis, and exhibits low rates in the development of resistance compared to antibiotics. ${ }^{5}$ Moreover, the synergistic effect of gallium with antibiotics was also observed. The therapeutic effects of $\mathrm{Ga}(\mathrm{III})$ are attributable to its identical chemical properties to $\mathrm{Fe}(\mathrm{III})$, being incorporated into essential $\mathrm{Fe}(\mathrm{III})-$ binding bacterial proteins/enzymes and leading to the disruption of various $\mathrm{Fe}$ (III)-dependent functions due to the fact that $\mathrm{Ga}(\mathrm{III})$ is unable to be reduced under physiological conditions. ${ }^{13-15}$ However, there appears to be a lack of studies at molecular levels on the mode of action of $\mathrm{Ga}(\mathrm{III})$-based antimicrobials. Knowledge on the key molecular targets of gallium and the bacterial cellular response under the stress of gallium might allow its efficacy to be further improved. 
Accumulated studies reported that the metabolic state of a bacterial cell could affect its susceptibility to antibiotics. ${ }^{16-18}$ Thus, sensitization of resistant bacteria to antibiotic treatment through metabolic stimuli represents a novel strategy to combat antimicrobial resistance. ${ }^{\mathbf{1 9 2 0}}$ Comprehensive analysis of cellular response of a bacterium to drug treatment through diversified approaches such as proteomics and functional metabolomics provides an insight into the mechanism of action of a drug, which may lead to enhanced therapeutic efficacy.

Herein, we report the identification of RNA polymerase as a key protein target that defines the bacteriostatic property of $\mathrm{Ga}(\mathrm{III})$ in $P$. aeruginosa. A functional metabolomics study of $P$. aeruginosa in response to $\mathrm{Ga}(\mathrm{III})$ treatment enabled acetate to be identified as the most effective metabolite that could enhance the bacteriostatic efficacy of $\mathrm{Ga}(\mathrm{III})$ against $P$. aeruginosa persisters, and the therapeutic effectiveness of co-therapy of $\mathrm{Ga}(\mathrm{III})$ and acetate was validated in mammalian cell infection and murine infection models.

\section{Results}

\section{Gallium targets bacterial RNA polymerase and attenuates transcription}

We first used the Ga-coordinated fluorescent probe $\mathrm{Ga}(\mathrm{III})-$ $T R A C E R^{21,22}$ to track Ga-binding proteins in live $P$. aeruginosa. The ability of $\mathrm{Ga}(\mathrm{III})-T R A C E R$ to label Ga-binding proteins in vitro was demonstrated using apo-lactoferrin (hLF) as shown in Fig. S1A (ESI $\dagger$ ). Upon incubation of $P$. aeruginosa cells with $\mathrm{Ga}(\mathrm{III})-T R A C E R$ and then irradiation with $\mathrm{UV}$ at $365 \mathrm{~nm}$ for $15 \mathrm{~min}$, we observed intense blue fluorescence throughout the
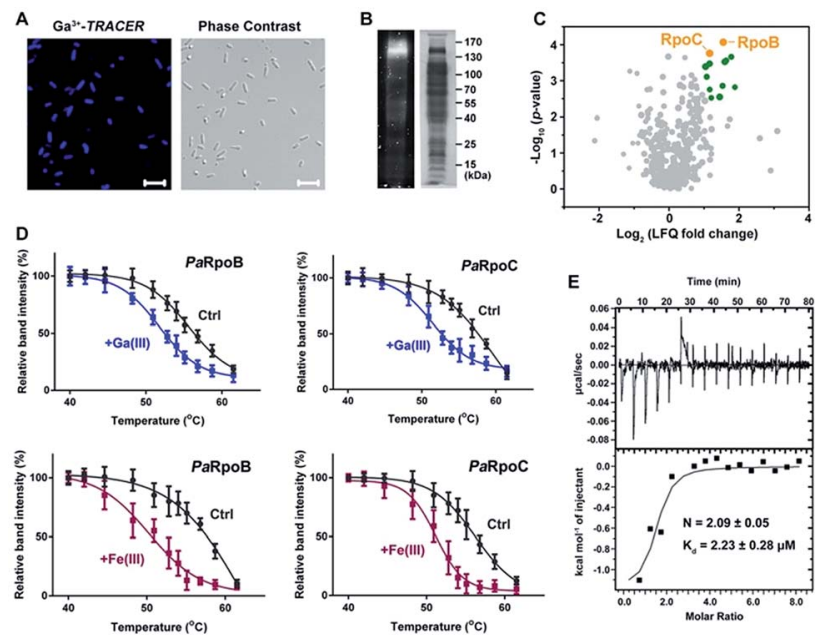

Fig. 1 Identification and validation of Ga-binding proteins in $P$. aeruginosa. (A) Confocal imaging of $P$. aeruginosa cells treated with $\mathrm{Ga}(\mathrm{II})-$ TRACER. (B) SDS-PAGE separation of $P$. aeruginosa cell lysate showing Ga(III)-TRACER-labelled fluorescent proteins. (C) Volcano plot showing the fold change and significance of protein intensities detected in the competitive Ga-IMAC experiment. Nodes in green color represent proteins with fold change $>2$ and $p$-value (of fold change) $<0.01$. (D) Cellular thermal shift analysis of $P a R p o B$ and $P a R-$ poC in intact $P$. aeruginosa cells treated with $\mathrm{Ga}\left(\mathrm{NO}_{3}\right)_{3}$ or $\mathrm{Fe}\left(\mathrm{NO}_{3}\right)_{3}$. (E) Calorimetric titration of gallium citrate $(1 \mathrm{mM})$ to apo-PaRpoB $(20 \mu \mathrm{M})$. pathogen (Fig. 1A), suggesting that the probe entered $P$. aeruginosa cells and labelled intracellular Ga-binding proteins. The cells were then lysed and separated by SDS-PAGE, and intense fluorescent bands at a molecular weight of 130-170 kDa were observed (Fig. 1B). Through peptide mass fingerprinting analysis, two proteins $P a$ RpoB and $P a$ RpoC of $c a$. $150 \mathrm{kDa}$ in accordance with the observed protein mass on the gel were identified as putative Ga-binding proteins (Table S1 $\dagger$ ).

We also employed competitive gallium-based immobilized metal affinity chromatography (Ga-IMAC) to identify Gabinding proteins in $P$. aeruginosa. Bacterial cell lysates either pre-treated or un-treated with $\mathrm{Ga}\left(\mathrm{NO}_{3}\right)_{3}$ were incubated with $\mathrm{Ga}$ IMAC resin. Pre-treatment of $\mathrm{Ga}\left(\mathrm{NO}_{3}\right)_{3}$ led to pre-saturation of the actual binding sites of the target proteins and resulted in reduced abundance in the eluted protein fraction. Over 200 proteins with at least two unique peptides were identified from the Ga-IMAC experiment. Through competitive analysis, PaRpoB and $P a$ RpoC were identified among the proteins with high enrichment factors (Fig. 1C). To validate the proteins identified by Ga-IMAC, a number of genes encoding proteins with high scores were cloned and proteins were overexpressed in $E$. coli cells, followed by supplementation of $\mathrm{Ga}$ (III)-TRACER to the cells. Only E. coli cells with $P a$ RpoB and $P a$ RpoC overexpressed could be fluorescent-labelled by the probe (Fig. S1B $\dagger$ ), further corroborating the binding of $\mathrm{Ga}(\mathrm{III})$ to $\mathrm{PaRpoB} / \mathrm{C}$ in cells.

The binding of $\mathrm{Ga}(\mathrm{III})$ to $\mathrm{PaRpoB} / \mathrm{C}$ in cells was further examined with a cellular thermal shift assay (CETSA) ${ }^{23}$ using the $\mathrm{PaRpoB} / \mathrm{C}$ polyclonal antibodies from $\mathrm{PaRpoB} / \mathrm{C}$-immunized rabbits. Addition of $\mathrm{Ga}\left(\mathrm{NO}_{3}\right)_{3}$ to the culture medium resulted in changes in the protein thermal stabilities, i.e., $T_{\mathrm{m}}$ from 57.2 to $53.5{ }^{\circ} \mathrm{C}$ and 58.0 to $52.3{ }^{\circ} \mathrm{C}$ for $P a$ RpoB and $P a$ RpoC, respectively, indicative of reduced protein thermal stabilities upon $\mathrm{Ga}$ (III) binding (Fig. 1D and $\mathrm{S} 1 \mathrm{C} \dagger$ ). Such changes were also observed in the presence of $\mathrm{Fe}\left(\mathrm{NO}_{3}\right)_{3}$ (Fig. 1D and $\mathrm{S} 1 \mathrm{C}^{\dagger}+$ ), demonstrating that $\mathrm{Fe}(\mathrm{III})$ also binds to $\mathrm{PaRpoB} / \mathrm{C}$ in cells. We further purified $P a \mathrm{RpoB} / \mathrm{C}$ proteins and investigated their Gabinding properties in vitro. A time dependent increase in fluorescence was observed upon mixing $P a$ RpoB with $\mathrm{Ga}$ (III)TRACER, leading to $c a$. 2-fold fluorescence enhancement (Fig. S1D $\dagger$ ). An isothermal titration calorimetry (ITC) experiment was then performed to monitor the titration of gallium citrate into EDTA-treated $\mathrm{PaRpoB} / \mathrm{C}$. The binding of gallium to apo- $P a \mathrm{RpoB} / \mathrm{C}$ resulted in a typical S-shaped curve, while presaturation of $P a$ RpoB with $\mathrm{Fe}(\mathrm{III})$ had no detectable Gabinding, indicating that $\mathrm{Ga}$ (III) shares common binding sites on the protein with Fe(III) (Fig. 1E and S2 $\dagger$ ). By fitting the data with a one-set-of-sites binding model, the dissociation constants were determined to be $2.23 \pm 0.28 \mu \mathrm{M}$ and $1.68 \pm 0.08$ $\mu \mathrm{M}$, with a stoichiometry of $2.09 \pm 0.05$ and $2.24 \pm 0.23$ for $\mathrm{Ga}$ (III) binding to $P a \mathrm{RpoB}$ and $P a \mathrm{RpoC}$, respectively. The binding stoichiometries are consistent with those obtained from ICP-MS analysis $(2.15 \pm 0.18$ and $2.11 \pm 0.26$ for Ga$P a$ RpoB and Ga-PaRpoC, respectively).

RpoB and RpoC are two subunits of DNA-dependent RNA polymerase, the key enzyme in the regulation of transcription and gene expression in living organisms. ${ }^{24}$ As Ga(III) binds to RpoB and RpoC both in vitro and in vivo, we hypothesize that the 
function of RNA polymerase could be interfered by Ga(III). To investigate the effect of $\mathrm{Ga}(\mathrm{III})$ on bacterial transcription, $P$. aeruginosa cells were pulse-labelled with 5-ethynyluridine (EU) for $4 \mathrm{~h}$ in the presence of $\mathrm{Ga}\left(\mathrm{NO}_{3}\right)_{3}$, followed by purification of the labelled nascent mRNA via click chemistry. Analysis of EUlabelled mRNA levels shows a global decrease in all analyzed $P$. aeruginosa house-keeping genes ${ }^{25}$ upon $\mathrm{Ga}(\mathrm{III})$ treatment (Fig. 2A), confirming the general defect in RNA polymerasemediated transcription in the presence of $\mathrm{Ga}$ (III). The bacteriostatic antibiotic rifamycin, a well-known RNA polymerase inhibitor that is able to suppress nascent mRNA levels in $P$. aeruginosa as evaluated by the same method (Fig. S3A $\dagger$ ), was employed as a positive control for comparing the effect of $\mathrm{Ga}$ (III). Interestingly, we found that bacterial growth is positively correlated with the levels of transcription regulated by $\mathrm{Ga}$ (III) and Fe(III). Supplementation of Ga(III) effectively inhibited the growth of $P$. aeruginosa in a dose-dependent manner. In contrast, Fe(III) obviously promoted bacterial growth (Fig. 2B), accompanied by enhanced bacterial transcription as revealed by the increased nascent mRNA levels (Fig. 2A). Given that uracil is an essential nucleobase for RNA synthesis, changes in its relative abundance were found to be correlated with the transcription inhibition or promotion mediated by $\mathrm{Ga}$ (III) or $\mathrm{Fe}(\mathrm{III})$, respectively (Fig. 2C).

\section{$\mathrm{Ga}(\mathrm{III})$ reduces TCA cycle flow and decreases bacterial respiration}

We then investigated the inhibition mechanisms of Ga(III) on $P$. aeruginosa at the transcriptome level. Pathway enrichment
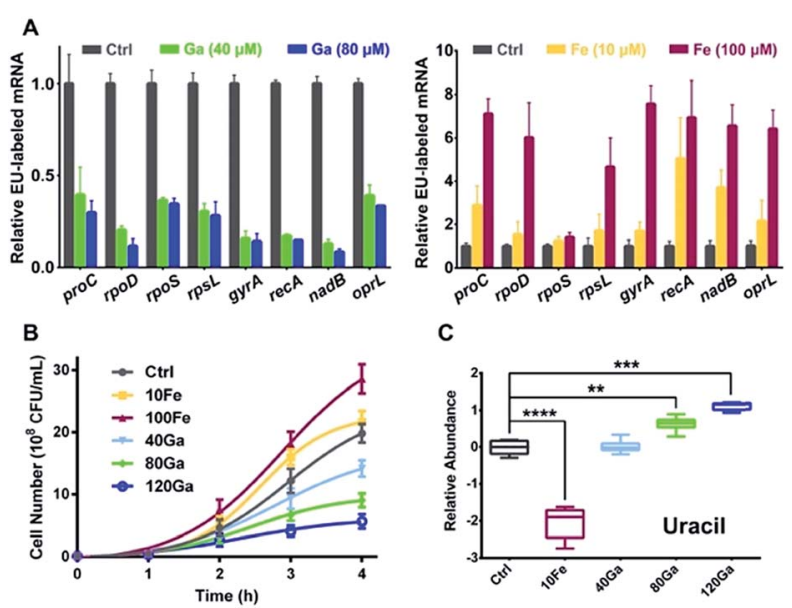

C

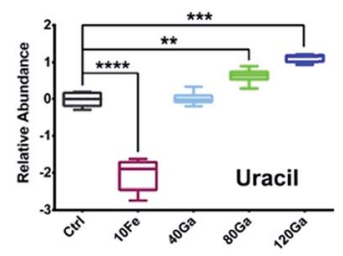

Fig. 2 Influence of $\mathrm{Ga}(\mathrm{II})$ or Fe(III) on $P$. aeruginosa transcription is positively correlated with bacterial growth. (A) Analysis of EU-labelled mRNA levels from $P$. aeruginosa cells treated with or without different concentrations of $\mathrm{Ga}$ (III) or Fe(III). (B) Growth curves and (C) relative abundance of uracil in $P$. aeruginosa under different $\mathrm{Ga}\left({ }^{\prime \prime I}\right)$ and $\mathrm{Fe}(\mathrm{II})$ treatment conditions. Data are presented as relative concentration values from biological replicates. The asterisks indicate significant difference from the control group $(* *, 0.001<p<0.01 ; * * *, 0.0001<p$ $<0.001$; and ****, $p<0.0001)$. The experimental groups correspond to untreated control (Ctrl), $10 \mu \mathrm{M} \mathrm{Fe(॥)} \mathrm{(10Fe),} 100 \mu \mathrm{M} \mathrm{Fe(॥)} \mathrm{(100Fe),} 10$

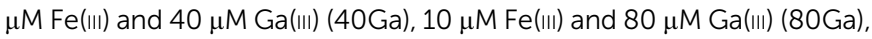
$10 \mu \mathrm{M}$ Fe(III) and $120 \mu \mathrm{M} \mathrm{Ga}(\mathrm{II})$ (120Ga) treatment conditions. analysis of the differentially regulated genes by $\mathrm{Ga}$ (III) indicates that the TCA cycle and oxidative phosphorylation are the pathways significantly influenced (Fig. S4A $\dagger$ ). We therefore evaluated the effect of $\mathrm{Ga}(\mathrm{III})$ on the bacterial central carbon metabolism. We found general dose-dependent decreases in the activities of TCA cycle enzymes upon Ga(III) treatment (Fig. S5A $\dagger$ ), and the enhanced inhibitory effect of Ga(III) against $P$. aeruginosa with the supplement of malonate, an inhibitor of succinate dehydrogenase (Fig. S5B $\dagger$ ), verifying that $\mathrm{Ga}$ (III) inhibits TCA cycle activity.

Oxygen functions as the terminal electron acceptor for aerobic respiration in the electron transport chain (ETC), and oxygen consumption thus serves as a useful indicator for ETC activity. ${ }^{26}$ We then quantified the oxygen consumption rate (OCR) of $P$. aeruginosa, and observed a linear increase in the OCR over time in the bacterium without treatment (as a control). However, the increase in the OCR was progressively suppressed with the supplementation of increasing amounts of $\mathrm{Ga}(\mathrm{III})$, and reduction in the OCR was observed at $256 \mu \mathrm{M} \mathrm{Ga}$ (Fig. S5C $\dagger$ ), indicating that $\mathrm{Ga}(\mathrm{III})$ inhibits respiratory activity. This was further validated by the observed enhanced inhibitory effect of $\mathrm{Ga}$ (III) upon supplementation of the cells with the ETC inhibitor $\mathrm{NaN}_{3}$, a cytochrome c oxidase inhibitor (Fig. S5D $\dagger$ ).

\section{Exogenous metabolites tune the susceptibility of $\boldsymbol{P}$. aeruginosa to $\mathrm{Ga}$ (III)}

To further understand the antimicrobial mechanisms of $\mathrm{Ga}(\mathrm{III})$ against $P$. aeruginosa, we performed a GC-MS-based metabolomics study to analyze the changes in metabolite abundance upon $\mathrm{Ga}(\mathrm{III})$ treatment. Overall, 62 metabolites with reliable signals were detected from $P$. aeruginosa metabolite extracts (Fig. 3A and Table S3†), and the PCA score plots showed clear separations of the metabolic profiles of different treatment groups (Fig. S6A $\dagger$ ). The differentially regulated metabolites were identified by one-way ANOVA analysis with significance values of $p<0.01$ (Table $\mathrm{S} 3 \dagger$ ).

After $4 \mathrm{~h}$ treatment, an increase in abundance of the majority of metabolites was observed in the group treated with a high concentration of $\mathrm{Ga}(\mathrm{III})(120 \mu \mathrm{M})$, including several essential amino acids (isoleucine, proline, valine, tyrosine and alanine) and the TCA cycle intermediates (succinic acid, citric acid, fumaric acid and $\alpha$-ketoglutaric acid) (Fig. 3A, S6B and $57 \dagger$ ), accompanied by increased cellular ATP levels (Fig. S6C $\dagger$ ). The accumulation of various metabolites shows the reduced metabolic rates and energy utilization in $P$. aeruginosa, which might be ascribed to the transcription suppression mediated growth inhibition of $P$. aeruginosa after treatment with Ga(III). Comparing the metabolic profiles of $\mathrm{Ga}$ (III)-treated groups with that of Fe(III)-deficient group (control group, Fig. 3A), although $\mathrm{Ga}$ (III) supplementation resulted in decreased bacterial $\mathrm{Fe}(\mathrm{III})$ accumulation (Fig. S6D $\dagger$ ), the changes in metabolite abundance upon $\mathrm{Ga}(\mathrm{III})$ treatment are mostly attributed to the addition of $\mathrm{Ga}$ (III) per se, and to a less extent to Ga-induced Fe(III) deficiency.

Metabolic response represents a strategy of cells to cope with external stimuli, which could be harnessed to regulate the metabolic status of cells and improve drug efficacy as reported 

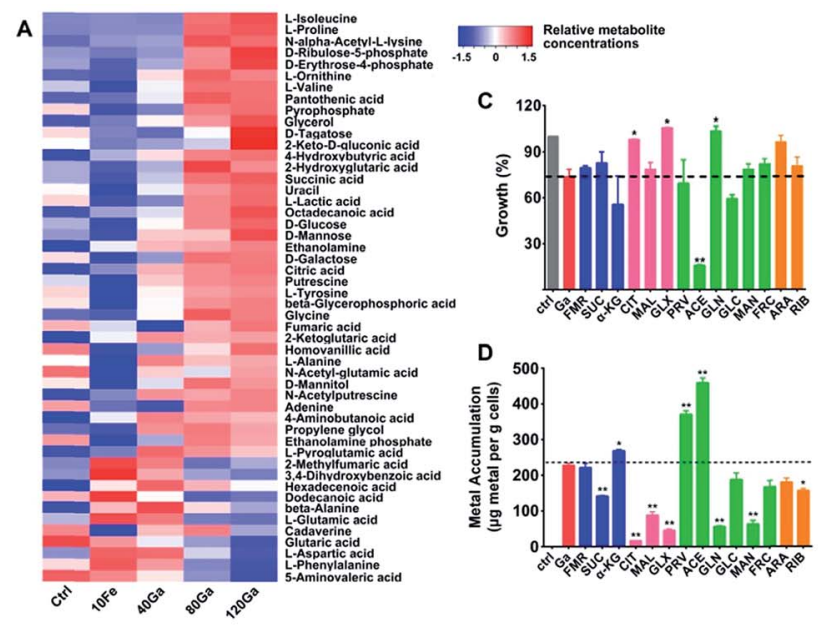

B

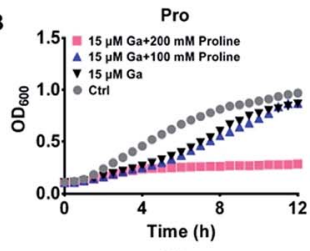

Glu
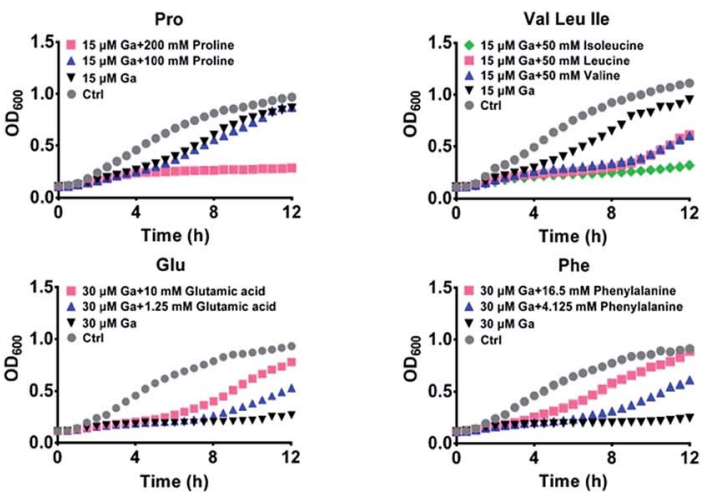

Fig. 3 Effect of exogenous metabolites on the susceptibility of $P$ aeruginosa to Ga(III) treatment. (A) Heat map for relative abundance of differentially regulated metabolites in $P$. aeruginosa upon $\mathrm{Ga}\left({ }^{\prime I I}\right)$ and Fe(III) treatment. The experimental groups correspond to untreated control (Ctrl), $10 \mu \mathrm{M} \mathrm{Fe}(I I I)$ (10Fe), $10 \mu \mathrm{M} \mathrm{Fe}(I I)$ and $40 \mu \mathrm{M} \mathrm{Ga(III)}(40 \mathrm{Ga})$, $10 \mu \mathrm{M}$ Fe(III) and $80 \mu \mathrm{M}$ Ga(III) (80Ga), $10 \mu \mathrm{M}$ Fe(III) and $120 \mu \mathrm{M} \mathrm{Ga(II)}$ $(120 \mathrm{Ga})$ treatment conditions. (B) Growth curves of $P$. aeruginosa in the presence of $\mathrm{Ga}$ (III) and Ga-regulated amino acids. Data show one representative result of three independent experiments. (C) Growth of $P$. aeruginosa in the presence of $G a(I I I)$ and various central carbon metabolites. (D) Metabolite-mediated accumulation of gallium in $P$. aeruginosa. Supplemented central carbon metabolites are as follows (sodium salts): fumarate (FMR), succinate (SUC), $\alpha$-ketoglutarate $(\alpha$ $K G)$, citrate $(C I T)$, malate (MAL), glyoxylate (GLX), pyruvate (PRV), acetate (ACE), gluconate $(G L N)$, glucose $(G L C)$, mannitol (MAN), fructose (FRC), arabinose (ARA), and ribose (RIB). Data are presented as mean \pm SEM from biological replicates. The asterisks indicate significant difference from the Ga(III) treatment group $(*, 0.01<p<0.05$ and $* *, 0.001<p<0.01$ )

in recent years. ${ }^{\mathbf{1 6 , 1 7 , 1 9 , 2 7}}$ We then examined whether metabolic stimulations in $P$. aeruginosa would modulate the inhibitory activity of $\mathrm{Ga}$ (III). As revealed by the metabolomics results, $\mathrm{Ga}$ (III) treatment led to significant changes in the levels of several essential amino acids, i.e., the up-regulated isoleucine, proline, and valine, and the down-regulated phenylalanine, aspartic acid, and glutamic acid. We first examined the bacterial growth upon $\mathrm{Ga}(\mathrm{III})$ treatment with or without the exogenous addition of the Ga-regulated amino acids. As shown in Fig. 3B, supplementation of the up-regulated amino acids proline and the branched-chain amino acids including isoleucine, leucine and valine to the bacterial culture media suppressed bacterial growth. In contrast, addition of the down-regulated amino acids such as glutamic acid and phenylalanine promoted bacterial growth, indicating that the inhibitory activity of $\mathrm{Ga}(\mathrm{III})$ could be regulated by external metabolic stimuli.

Given that our combined study at transcriptomics and metabolomics levels showed that the central carbon metabolism of $P$. aeruginosa was significantly influenced by Ga(III), we therefore screened carbon source metabolites covering various central metabolic pathways, i.e., the TCA cycle, pyruvate metabolism and glycolysis. We found that these metabolites exhibited different effects on the susceptibility of $P$. aeruginosa to $\mathrm{Ga}(\mathrm{III})$, as revealed by the $12 \mathrm{~h}$ growth curves of the bacterium under different treatment conditions (Fig. 3C and S8A $\dagger$ ). In the presence of $\mathrm{Ga}(\mathrm{III})$, addition of acetate significantly decreased bacterial growth by $80 \%$; while addition of citrate, glyoxylate and gluconate slightly promoted bacterial growth (Fig. 3C and $\mathrm{S} 8 \mathrm{~A} \dagger$ ). Citrate and glyoxylate were also found to be able to promote the growth of $P$. aeruginosa under the stress of high concentration $(256 \mu \mathrm{M})$ of $\mathrm{Ga}$ (III) (Fig. S8B $\dagger$ ). Addition of metabolites alone had no significant effect on bacterial growth (Fig. S8C $\dagger$ ). We then examined $\mathrm{Ga}(\mathrm{III})$ uptake by $P$. aeruginosa after co-treatment of metabolites with $\mathrm{Ga}$ (III), and found that the enhanced antimicrobial activity was positively correlated with the levels of Ga uptake (Fig. 3D). Acetate, which significantly inhibits bacterial growth, induced the uptake of high levels of Ga (2-fold), implying that the increased Ga uptake is responsible for the inhibited growth.

\section{Co-treatment of $\mathrm{Ga}$ (III) and acetate enhances the antimicrobial efficacy of $\mathrm{Ga}$ (III) in cell and murine infection models and reduces $P$. aeruginosa virulence}

As acetate was identified as the most effective metabolite in enhancing the inhibitory activity of $\mathrm{Ga}$ (III) on bacterial growth, we therefore evaluated the effectiveness of the co-therapy of $\mathrm{Ga}$ (III) and acetate. Enhanced antimicrobial activity of $\mathrm{Ga}$ (III) caused by acetate was also demonstrated on a clinically isolated $P$. aeruginosa strain (Fig. S9†). In CF patients with chronic antibiotic treatment, $P$. aeruginosa frequently exists as persister cells. $^{28}$ We first examined whether co-therapy of Ga(III) and acetate is effective on $P$. aeruginosa persister cells. The persister cells were isolated by treating stationary-phase cells with $10 \mu \mathrm{g}$ $\mathrm{mL}^{-1}$ ciprofloxacin or $10 \mu \mathrm{g} \mathrm{mL}^{-1}$ ofloxacin, and the survival cells were then exposed to $\mathrm{Ga}$ (III) alone or $\mathrm{Ga}$ (III) in combination with metabolites. The $24 \mathrm{~h}$ growth curves showed that Ga(III) (4 $\mu \mathrm{M}$ ) only slightly inhibited bacterial growth, while the combination of $\mathrm{Ga}$ (III) with acetate $(30 \mathrm{mM}$ ) could completely inhibit the growth of persister cells. In contrast, the combined use of $\mathrm{Ga}(\mathrm{III})$ with glyoxylate $(30 \mathrm{mM})$ promoted bacterial growth (Fig. 4A and B), consistent with the observations on exponentialphase $P$. aeruginosa.

We next examined the effects of co-administration of $\mathrm{Ga}(\mathrm{III})$ with acetate in a mammalian cell infection model. A549 cells were infected with $P$. aeruginosa at a multiplicity of infection (MOI) of 10 for $1 \mathrm{~h}$. The infected A549 cells were then exposed to $\mathrm{Ga}$ (III) or acetate alone or their combination for $24 \mathrm{~h}$, and the 

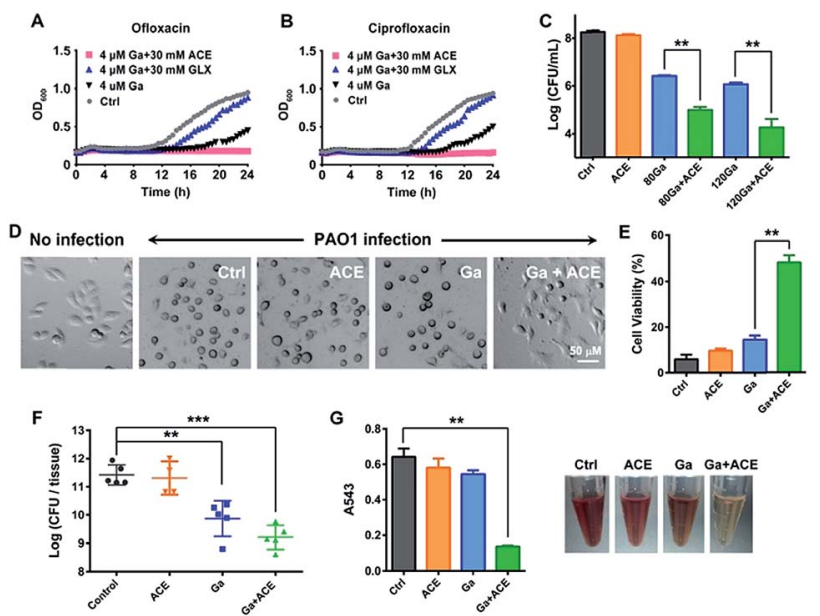

Fig. 4 Acetate enhances the antimicrobial effectiveness of Ga(III) and decreases $P$. aeruginosa virulence. (A and $B$ ) Growth curves of $P$. aeruginosa persisters in the presence of $\mathrm{Ga}$ (III) or the combination with acetate or glyoxylate. (C) A549 cell-associated bacterial colony counts in the in vitro cell infection model. (D) Cell morphology after $P$. aeruginosa infection for $4 \mathrm{~h}$. (E) Viability of A549 cells after $4 \mathrm{~h} P$. aeruginosa infection, corresponding to data in (D). (F) CFU of $P$. aeruginosa counted in the infected mice tissues after treatment with the vehicle, sodium acetate $\left(1 \mathrm{~g} \mathrm{~kg}^{-1}\right), \mathrm{Ga}\left(\mathrm{NO}_{3}\right)_{3}\left(2 \mathrm{mg} \mathrm{kg}^{-1}\right)$ or their combinations $48 \mathrm{~h}$ post-infection. (G) Haemolytic activities of $P$. aeruginosa exposed to sub-inhibitory concentrations of acetate $(10 \mathrm{mM}), \mathrm{Ga}(\mathrm{III})(6 \mu \mathrm{M})$ or their combination. Data are presented as mean \pm SEM from biological replicates. The asterisks indicate significant difference from the Ctrl or Ga(III) treatment group $(* *, 0.001<p<0.01$ and $* * *, p<0.001)$.

cell-associated bacteria were enumerated by agar plating. Our results showed that the viable bacterial loads were at a level of $10^{8} \mathrm{CFU}$ when $30 \mathrm{mM}$ acetate was used, which is comparable to those of the untreated control group. The bacterial loads dropped to a level of $10^{6} \mathrm{CFU}$ when treated with $80-120 \mu \mathrm{M}$ $\mathrm{Ga}(\mathrm{III})$, and further dropped to a level of $10^{4} \mathrm{CFU}$ when $\mathrm{Ga}$ (III) $(120 \mu \mathrm{M})$ and acetate $(30 \mathrm{mM})$ were co-administered (Fig. 4C); however, the cells cultured under the stress of $\mathrm{Fe}(\mathrm{III})$ and acetate showed increased bacterial loads (Fig. S10†). Notably, we observed a marked cytoprotective effect on $P$. aeruginosa infected A549 cells by the combined Ga-acetate treatment. A549 cells were infected with $P$. aeruginosa in the presence of $\mathrm{Ga}$ (III) or acetate, or their combination, and the cytotoxic effect was studied after infection for $4 \mathrm{~h}$. The infected cells were found to have a round morphology in the control and single therapy groups, while the cell morphology remained almost unchanged before and after bacterial infection in the group with combined Ga-acetate treatment (Fig. 4D and S11†) and cell viability was also significantly increased by $34 \%$ compared with that of $\mathrm{Ga}(\mathrm{III})$ treatment alone (Fig. 4E).

We further evaluated the in vivo effectiveness of the $\mathrm{Ga}-$ acetate combination in a murine skin infection model. Female $\mathrm{BALB} / \mathrm{c}$ mice were infected with $5 \times 10^{6} \mathrm{CFU}$ of $P$. aeruginosa on an excisional skin wound. The infected mice received treatment of $\mathrm{Ga}(\mathrm{III})$ or the combination with acetate therapy twice daily, and the mice were sacrificed $48 \mathrm{~h}$ post-infection for colony counting. As shown in Fig. 4f, treatment with $\mathrm{Ga}$ (III) alone reduced the cell count by $c a$. 10-fold compared with that of the control group, whereas treatment with the $\mathrm{Ga}(\mathrm{III})$ and acetate combination further reduced the bacterial count by $c a$. 10-fold compared with that of the $\mathrm{Ga}$ (III) treatment group, clearly demonstrating that acetate can enhance the antimicrobial activity of $\mathrm{Ga}(\mathrm{III})$ in vivo. We then examined the effect of cotherapy of acetate and $\mathrm{Ga}(\mathrm{III})$ on the pathogenicity of $P$. aeruginosa. In comparison to $\mathrm{Ga}(\mathrm{III})$ or acetate treatment alone, the combination of $\mathrm{Ga}(\mathrm{III})$ and acetate at sub-inhibitory concentration significantly reduced bacterial hemolysin secretion in mammalian cells (Fig. 4G). Moreover, at higher drug concentrations ( $80 \mu \mathrm{M} \mathrm{Ga}$ (III) and $30 \mathrm{mM}$ acetate), the virulence factors secreted by the pathogen including elastase, protease, pyocyanin, pyoverdine, phospholipase $\mathrm{C}$, phamnolipid and exotoxin $\mathrm{A}$ were extensively down-regulated at transcriptome levels (Fig. S12A and Table S4†), implying reduced virulence of the bacterium.

\section{Combined Ga(III)-acetate treatment regulates P. aeruginosa metabolome}

We further investigated the mechanisms of action of co-therapy of $\mathrm{Ga}$ (III) and acetate at transcriptome and metabolome levels. Cluster analysis and principal component analysis (PCA) of RNA-seq and GC-MS data showed certain similarity between the control and acetate treatment groups; however, the $\mathrm{Ga}(\mathrm{III})$ and combined treatment groups were clearly separated from the control group (Fig. S12B and $\mathrm{C}^{\dagger}$ ), suggesting that $\mathrm{Ga}$ (III) is responsible for the observed antimicrobial effect. The combined $\mathrm{Ga}(\mathrm{III})$-acetate treatment further attenuated bacterial transcription as revealed by decreased mRNA levels (Fig. S3B $\dagger$ ).

To evaluate the effect of the combined treatment on TCA cycle flow, we performed a ${ }^{13} \mathrm{C}_{2}$-acetate NTFD (non-targeted tracer fate detection) experiment on $P$. aeruginosa in the absence and the presence of Ga(III) by GC-MS. ${ }^{29}$ Three ${ }^{13} \mathrm{C}$ labeled TCA cycle metabolites including citrate, succinate and fumarate were detected, suggesting that the exogenous acetate was converted by acetyl-CoA synthase to labelled acetyl-CoA and then fluxed to the TCA cycle. According to a previous study, the sum of M1 and M3 labels $\left(\nu_{\mathrm{TCA}}\right)$ refers to the turnover of a particular metabolite in the TCA cycle, while M2 plus M3 $\left(\nu_{\mathrm{ACE}}\right)$ is the flux of the labeled acetate to the TCA cycle, thus the $(\mathrm{M} 1+\mathrm{M} 3) /(\mathrm{M} 2+\mathrm{M} 3)$ ratio represents the relative flux for the certain metabolite in the TCA cycle. ${ }^{17}$ The observation of the decreases in $\nu_{\mathrm{TCA}} / \nu_{\mathrm{ACE}}$ ratios and abundance of the three metabolites in the TCA cycle under Ga(III) stress further verified the inhibition of the TCA cycle by Ga(III) (Fig. 5A). This was further corroborated by the reduced $[\mathrm{NADH}] /\left[\mathrm{NAD}^{+}\right]$ratio upon $\mathrm{Ga}(\mathrm{III})$ and the combined treatment (Fig. 5B), representing decreased reducing power generated through TCA cycle flow.

In addition to the ${ }^{13} \mathrm{C}$-labeled metabolites in the TCA cycle, a number of ${ }^{13} \mathrm{C}$-labeled amino acids, nucleotides and fatty acids were detected upon the co-treatment of $\mathrm{Ga}(\mathrm{III})$ and ${ }^{13} \mathrm{C}_{2}$ acetate (Fig. S13†), suggesting that a widespread metabolome reprogramming in $P$. aeruginosa occurred to deal with $\mathrm{Ga}(\mathrm{III})$ stress. Moreover, treatment of the bacterium with $\mathrm{Ga}$ (III) also resulted in the accumulation of glucose, and depletion of 

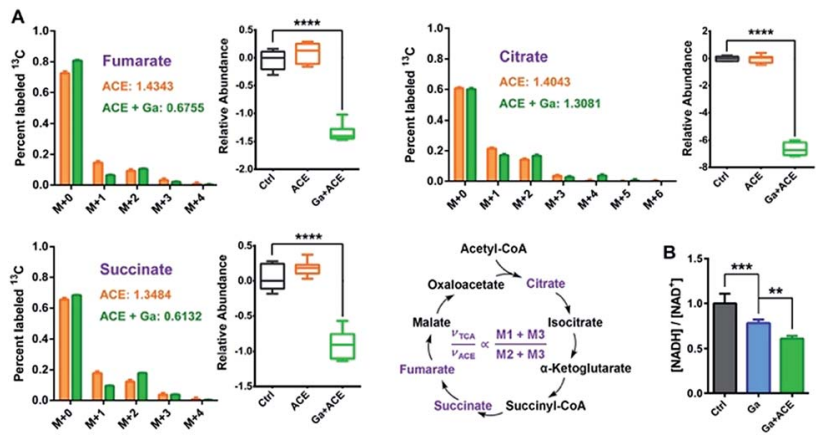

Fig. 5 Combined $\mathrm{Ga}(\mathrm{III})$ and acetate treatment leads to reduced TCA cycle flow. (A) Mass isotopomer distributions of ${ }^{13} \mathrm{C}_{2}$-labelled acetate detected in the TCA cycle and the relative abundances of cycle metabolites. (B) Ratios of bacterial cellular NADH and NAD ${ }^{+}$levels after treatment with $\mathrm{Ga}(\mathrm{III})$ and acetate. Data are presented as mean $\pm \mathrm{SEM}$ from biological replicates. The asterisks indicate significant difference from the $\mathrm{Ctrl}$ or $\mathrm{Ga}(\mathrm{III})$ treatment group $(* *, 0.001<p<0.01$ and $* * *, p<$ 0.001)

glucose 6-phosphate and ribose 5-phosphate (Fig. S14†), the key metabolites in glycolysis and the pentose phosphate pathway, indicative of suppressed glucose catabolism by $\mathrm{Ga}$ (III).

Transcriptome analysis showed that treatment of the bacterium with acetate led to marked regulation of a number of genes enriched in various amino acid metabolic pathways (Fig. S4B $\dagger$ ), in particular, the up-regulation of the phenylalanine and tyrosine metabolic pathway enriched by the significantly upregulated genes $h p d$, maiA, $h m g A, p h h A$ and $p h h C$ (Table S5†). Interestingly, proteins Hpd, PhhA and HmgA in this pathway were reported to bind ferric iron. ${ }^{30}$ To investigate the role of these proteins in the antimicrobial action of $\mathrm{Ga}$ (III), we generated mutant strains $\Delta p h h A, \Delta h m g A$ and $\Delta h p d$ by transposon mutagenesis and found that all the mutant strains showed reduced susceptibility towards $\mathrm{Ga}$ (III) in comparison to the wild type strain (Fig. 6A), indicating the involvement of these genes in mediating $\mathrm{Ga}(\mathrm{III})$ sensitivity in $P$. aeruginosa.

\section{Discussion}

Metal-based antimicrobials have received renewed interest in the era of antimicrobial resistance, which has led to the drying up of antibiotic pipelines. Recently, an FDA approved gallium drug (Ganite ${ }^{\circledR}$ ) has been shown to have therapeutic effects on mice and humans with lung infections from $P$. aeruginosa. ${ }^{5}$ Although it has long been known that $\mathrm{Ga}$ (III) can disrupt bacterial iron metabolism owing to its similarity to $\mathrm{Fe}(\mathrm{III})$, a better understanding of the mode of action of $\mathrm{Ga}$ (III) at molecular levels allows its therapeutic potential to be fully explored. Previously, Ga(III) has been demonstrated to interact with "bacterial transferrin", the periplasmic iron-transport protein, ${ }^{31}$ and hitA gene has been also identified to associate with $\mathrm{Ga}(\mathrm{III})$ resistance in $P$. aeruginosa. ${ }^{5,32}$ However, the $\Delta$ hitA mutant strain of $P$. aeruginosa still shows susceptibility towards $\mathrm{Ga}(\mathrm{III}),{ }^{5}$ strongly indicating the presence of key cellular targets of $\mathrm{Ga}(\mathrm{III})$, which have not been characterized. In the present study, by using metalloproteomics approaches, we uncovered a novel
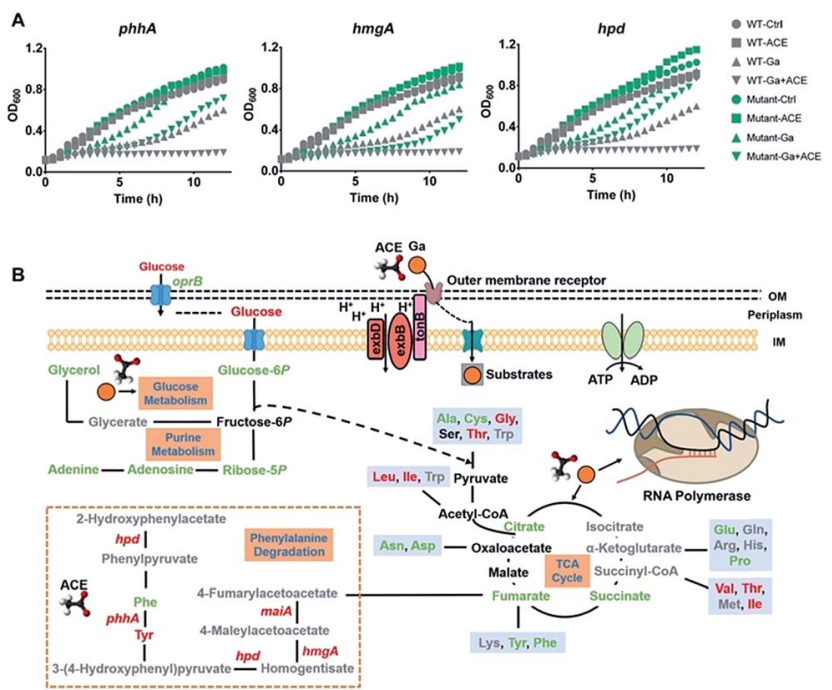

Fig. 6 Acetate enhances the bacteriostatic activity of Ga(III) and modulates bacterial metabolism. (A) Growth curves of wild type and transposon mutant $P$. aeruginosa strains exposed to Ga(II) $(20 \mu \mathrm{M})$ or acetate $(30 \mathrm{mM})$ or their combination. (B) Schematic representation of the changes in the transcriptome and metabolome of $P$. aeruginosa under the stress of $\mathrm{Ga}(\mathrm{III})$ and acetate, with green and red colors representing down-regulated and up-regulated metabolites/genes, respectively.

Ga(III) target, RNA polymerase (RNAP), in $P$. aeruginosa. We found that $\mathrm{Ga}$ (III) bound to two subunits RpoB and RpoC of the core RNAP enzyme with a stoichiometry of $2: 1$, and Fe(III) was also able to bind to the two proteins and interfered with the $\mathrm{Ga}(\mathrm{III})$ binding ability.

RNAP is the basic enzyme in transcription and is well characterized to be a target of the bacteriostatic antibiotics rifamycins. $^{24,33} \mathrm{Mg}(\mathrm{II}), \mathrm{Zn}$ (II) and the [4Fe-4S] cluster have been reported to be the cofactors of RNAP. ${ }^{34}$ As an essential process for bacterial growth and survival, transcription is the first step of gene expression carried out by RNAP, and there exist exquisite crosstalks between transcription and metabolism to regulate cellular enzyme levels. ${ }^{35,36}$ Indeed, we observed a positive correlation between $\mathrm{Fe}(\mathrm{III})$ - and $\mathrm{Ga}(\mathrm{III})$-mediated bacterial growth and transcriptional regulation (Fig. 2). As a bacteriostatic antimicrobial, $\mathrm{Ga}(\mathrm{III})$ markedly inhibited $P$. aeruginosa growth as well as transcription processes by targeting RpoB and RpoC, evidenced by the decreased nascent mRNA levels and a significant accumulation of the nucleobase uracil owing to reduced amounts incorporated into RNA chains. In contrast, the presence of $\mathrm{Fe}(\mathrm{III})$ was found to promote bacterial growth and transcription processes. However, how metal-mediated transcriptional regulation may inhibit or promote bacterial growth remains unclear. Phenotypically, Ga(III) treatment led to ATP accumulation, OCR reduction, undetectable ROS elevation and central carbon metabolism suppression featured by reduced TCA cycle flow and decreased glucose consumption, which are consistent with the phenotypic features of bacteria in response to bacteriostatic antibiotics as reported previously. ${ }^{26}$ Therefore, upon $\mathrm{Ga}(\mathrm{III})$ treatment, $P$. aeruginosa may enter 
a metabolically quiescent state as a result of drug-target interaction to withstand $\mathrm{Ga}$ (III) stress. We thus believe that targeting bacterial RNAP may define the bacteriostatic feature of the antimicrobial $\mathrm{Ga}$ (III), and such bacteriostatic agents are as effective as bactericidal agents in the treatment of bacterial infections clinically. ${ }^{37}$

The metabolome holds precise information on a cell's status, and offers a rich resource for the manipulation of the cell's function and vulnerability. For instance, regulations in bacterial metabolism could alter aminoglycoside sensitivity in bacterial persisters, stationary-phase bacteria, and antibiotic-resistant bacteria; ${ }^{16-18}$ L-arginine could directly impact the metabolic fitness and enhance the anti-tumor activity of central memory $\mathrm{T}$ cells. ${ }^{38}$ Herein, we show that the inhibitory effect of Ga(III) could be manipulated by exogenous metabolic stimuli, and supplementation of the metabolites that are down-regulated or upregulated by $\mathrm{Ga}$ (III) resulted in $\mathrm{Ga}$ (III) activity being suppressed or enhanced, indicating that the efficacy of a drug could be modulated according to the drug-induced metabolome changes. Our results further reveal that the central carbon metabolite acetate effectively increased $\mathrm{Ga}$ (III) uptake by $P$. aeruginosa, leading to enhanced bacteriostatic activity as demonstrated on persister cells, and A549 cell infection and murine skin infection models. Moreover, at transcriptome levels, acetate activated the Fe-demanding phenylalanine degradation pathway, and the transposon mutations of key genes in this pathway made the bacterium less sensitive to $\mathrm{Ga}(\mathrm{III})$, which may also contribute to acetate-induced enhancement of $\mathrm{Ga}$ (III) antimicrobial activity. Given that acetic acid has already been used clinically for the treatment of infected burn wounds, ${ }^{39,40}$ the combination of acetate with an FDA approved gallium drug may provide a rapid and cost-effective route to new therapeutic strategies to cope with the current crisis of antibiotic resistance in clinics.

\section{Conclusions}

The rapid emergence and spread of antibiotic resistant bacterial strains and the diminished antibiotic pipelines have led to renewed interest in using metal-based antimicrobials for fighting against infectious diseases in clinics. Knowledge of the mechanisms of action of these antimicrobials allows us to further enhance their effectiveness or to rationally design more potent antimicrobial agents. Ga(III) has broad-spectrum antimicrobial activity against a range of infectious pathogens and shows evidence to be a safe and effective antimicrobial in the treatment of human lung infections in clinical trials. By integration of transcriptomics, proteomics and metabolomics, we show for the first time that $\mathrm{Ga}$ (III) targets bacterial transcription to inhibit cell growth, which defines the bacteriostatic feature of Ga(III) against $P$. aeruginosa (Fig. 6B). Moreover, we identified that the central carbon metabolite acetate could effectively enhance the bacteriostatic activity of $\mathrm{Ga}$ (III) through increased uptake and activated the Fe-demanding genes in the phenylalanine degradation pathway (Fig. 6B). The combination of acetate and $\mathrm{Ga}$ (III) enabled eradication of $P$. aeruginosa persister cells, and the increased antimicrobial activity of the co-therapy was further demonstrated in mammalian cell and murine infection models. Our results provide novel insights into the modes of action of $\mathrm{Ga}(\mathrm{III})$, as well as strategies for the development of efficient Ga-based therapies.

\section{Conflicts of interest}

There are no conflicts to declare.

\section{Acknowledgements}

This work was supported by the National Natural Science Foundation of China (21601209 and 21671203), the Natural Science Foundation of Guangdong Province (2017A030313063), the Research Grants Council of Hong Kong (17333616P and 17307017P), the Fundamental Research Funds for the Central Universities, a starting fund from Sun Yat-sen University, and the University of Hong Kong. We thank Dr Zifeng Wang for the suggestions on transcription assay design; Dr Minji Wang for the information on transposon mutant $P$. aeruginosa strains; Dr Hui Zhang and Wenhua Lu for the assistance in the Seahorse Experiment.

\section{Notes and references}

1 R. Wang, T.-P. Lai, P. Gao, H. Zhang, P.-L. Ho, P. C.-Y. Woo, G. Ma, R. Y.-T. Kao, H. Li and H. Sun, Nat. Commun., 2018, 9, 439, DOI: 10.1038/s41467-018-02828-6.

2 Y. Wang, L. Hu, F. Xu, Q. Quan, Y.-T. Lai, W. Xia, Y. Yang, Y.-Y. Chang, X. Yang, Z. Chai, J. Wang, I. K. Chu, H. Li and H. Sun, Chem. Sci., 2017, 8, 4626-4633.

3 J. R. Morones-Ramirez, J. A. Winkler, C. S. Spina and J. J. Collins, Sci. Transl. Med., 2013, 5, 190ra181, DOI: 10.1126/scitranslmed.3006276.

4 M. B. Harbut, C. Vilcheze, X. Luo, M. E. Hensler, H. Guo, B. Yang, A. K. Chatterjee, V. Nizet, W. R. Jacobs Jr, P. G. Schultz and F. Wang, Proc. Natl. Acad. Sci. U. S. A., 2015, 112, 4453-4458.

5 C. H. Goss, Y. Kaneko, L. Khuu, G. D. Anderson, S. Ravishankar, M. L. Aitken, N. Lechtzin, G. Zhou, D. M. Czyz, K. McLean, O. Olakanmi, H. A. Shuman, M. Teresi, E. Wilhelm, E. Caldwell, S. J. Salipante, D. B. Hornick, R. J. Siehnel, L. Becker, B. E. Britigan and P. K. Singh, Sci. Transl. Med., 2018, 10, eaat7520, DOI: 10.1126/scitranslmed.aat7520.

6 J. A. Lemire, J. J. Harrison and R. J. Turner, Nat. Rev. Microbiol., 2013, 11, 371-384.

7 T. S. Cohen and A. Prince, Nat. Med., 2012, 18, 509-519.

8 M. W. Azam and A. U. Khan, Drug Discovery Today, 2019, 24, 350-359.

9 K. Poole, Front. Microbiol., 2011, 2, 65, DOI: 10.3389/ fmicb.2011.00065.

10 C. Bonchi, F. Imperi, F. Minandri, P. Visca and E. Frangipani, BioFactors, 2014, 40, 303-312.

11 S. Hijazi, P. Visca and E. Frangipani, Front. Cell. Infect. Microbiol., 2017, 7, 12, DOI: 10.3389/fcimb.2017.00012. 
12 E. Banin, A. Lozinski, K. M. Brady, E. Berenshtein, P. W. Butterfield, M. Moshe, M. Chevion, E. P. Greenberg and E. Banin, Proc. Natl. Acad. Sci. U. S. A., 2008, 105, 16761-16766.

13 C. R. Chitambar, Biochim. Biophys. Acta, 2016, 1863, 20442053.

14 F. Minandri, C. Bonchi, E. Frangipani, F. Imperi and P. Visca, Future Microbiol., 2014, 9, 379-397.

15 Y. Kaneko, M. Thoendel, O. Olakanmi, B. E. Britigan and P. K. Singh, J. Clin. Invest., 2007, 117, 877-888.

16 K. R. Allison, M. P. Brynildsen and J. J. Collins, Nature, 2011, 473, 216-220.

17 B. Peng, Y. B. Su, H. Li, Y. Han, C. Guo, Y. M. Tian and X. X. Peng, Cell Metab., 2015, 21, 249-261.

18 S. Meylan, C. B. Porter, J. H. Yang, P. Belenky, A. Gutierrez, M. A. Lobritz, J. Park, S. H. Kim, S. M. Moskowitz and J. J. Collins, Cell Chem. Biol., 2017, 24, 195-206.

19 B. Peng, H. Li and X. X. Peng, Protein Cell, 2015, 6, 628-637.

20 S. Meylan, I. W. Andrews and J. J. Collins, Cell, 2018, 172, 1228-1238.

21 Y.-T. Lai, Y.-Y. Chang, L. Hu, Y. Yang, A. Chao, Z.-Y. Du, J. A. Tanner, M.-L. Chye, C. Qian, K.-M. Ng, H. Li and H. Sun, Proc. Natl. Acad. Sci. U. S. A., 2015, 112, 2948-2953.

22 Y. T. Lai, Y. Yang, L. Hu, T. Cheng, Y. Y. Chang, M. KoohiMoghadam, Y. Wang, J. Xia, J. Wang, H. Li and H. Sun, Metallomics, 2017, 9, 38-47.

23 D. M. Molina, R. Jafari, M. Ignatushchenko, T. Seki, E. A. Larsson, C. Dan, L. Sreekumar, Y. Cao and P. Nordlund, Science, 2013, 341, 84-87.

24 S. Borukhov and E. Nudler, Trends Microbiol., 2008, 16, 126134.

25 B. Alqarni, B. Colley, J. Klebensberger, D. McDougald and S. A. Rice, J. Microbiol. Methods, 2016, 127, 182-187.

26 M. A. Lobritz, P. Belenky, C. B. Porter, A. Gutierrez, J. H. Yang, E. G. Schwarz, D. J. Dwyer, A. S. Khalil and
J. J. Collins, Proc. Natl. Acad. Sci. U. S. A., 2015, 112, 81738180.

27 Y. B. Su, B. Peng, H. Li, Z. X. Cheng, T. T. Zhang, J. X. Zhu, D. Li, M. Y. Li, J. Z. Ye, C. C. Du, S. Zhang, X. L. Zhao, M. J. Yang and X. X. Peng, Proc. Natl. Acad. Sci. U. S. A., 2018, 115, E1578-E1587.

28 R. A. Fisher, B. Gollan and S. Helaine, Nat. Rev. Microbiol., 2017, 15, 453-464.

29 K. Hiller, C. M. Metallo, J. K. Kelleher and G. Stephanopoulos, Anal. Chem., 2010, 82, 6621-6628.

30 C. The UniProt, Nucleic Acids Res., 2017, 45, D158-D169.

31 M. Guo, I. Harvey, W. Yang, L. Coghill, D. J. Campopiano, J. A. Parkinson, R. T. MacGillivray, W. R. Harris and P. J. Sadler, J. Biol. Chem., 2003, 278, 2490-2502.

32 R. Garcia-Contreras, E. Lira-Silva, R. Jasso-Chavez,

I. L. Hernandez-Gonzalez, T. Maeda, T. Hashimoto, F. C. Boogerd, L. Sheng, T. K. Wood and R. MorenoSanchez, Int. J. Med. Microbiol., 2013, 303, 574-582.

33 H. G. Floss and T.-W. Yu, Chem. Rev., 2005, 105, 621-632.

34 M. N. Wojtas, M. Mogni, O. Millet, S. D. Bell and

N. G. A. Abrescia, Nucleic Acids Res., 2012, 40, 9941-9952.

35 C. Ma, X. Yang and P. J. Lewis, Microbiol. Mol. Biol. Rev., 2016, 80, 139-160.

36 S. Donati, T. Sander and H. Link, Wiley Interdiscip. Rev.: Syst. Biol. Med., 2018, 10, e1396, DOI: 10.1002/wsbm.1396.

37 N. Wald-Dickler, P. Holtom and B. Spellberg, Clin. Infect. Dis., 2018, 66, 1470-1474.

38 R. Geiger, J. C. Rieckmann, T. Wolf, C. Basso, Y. Feng, T. Fuhrer, M. Kogadeeva, P. Picotti, F. Meissner, M. Mann, N. Zamboni, F. Sallusto and A. Lanzavecchia, Cell, 2016, 167, 829-842.

39 B. S. Nagoba, S. P. Selkar, B. J. Wadher and R. C. Gandhi, J. Infect. Public Health, 2013, 6, 410-415.

40 V. L. Madhusudhan, Int. Wound J., 2016, 13, 1129-1136. 